VLSI Design

1995, Vol. 3, No. 2, pp. 145-158

Reprints available directly from the publisher

Photocopying permitted by license only (c) 1995 OPA (Overseas Publishers Association)

Amsterdam B.V. Published under license by

Gordon and Breach Science Publishers SA

Printed in Malaysia

\title{
Mixed-RKDG Finite Element Methods for the 2-D Hydrodynamic Model for Semiconductor Device Simulation
}

\author{
ZHANGXIN CHEN, ${ }^{1}$ BERNARDO COCKBURN, ${ }^{2}$ JOSEPH W. JEROME, ${ }^{3}$ \\ CHI-WANG SHU ${ }^{4}$
}

(Received January 1, 1994)

\begin{abstract}
In this paper we introduce a new method for numerically solving the equations of the hydrodynamic model for semiconductor devices in two space dimensions. The method combines a standard mixed finite element method, used to obtain directly an approximation to the electric field, with the so-called Runge-Kutta Discontinuous Galerkin (RKDG) method, originally devised for numerically solving multi-dimensional hyperbolic systems of conservation laws, which is applied here to the convective part of the equations. Numerical simulations showing the performance of the new method are displayed, and the results compared with those obtained by using Essentially Nonoscillatory (ENO) finite difference schemes. From the perspective of device modeling, these methods are robust, since they are capable of encompassing broad parameter ranges, including those for which shock formation is possible. The simulations presented here are for Gallium Arsenide at room temperature, but we have tested them much more generally with considerable success.
\end{abstract}

Key Words: hydrodynamic model, MESFET, mixed methods, finite volume methods, finite element methods, conservation laws

\section{INTRODUCTION}

Tn this paper, we propose and test a new method for numerically solving the equations of the hydrodynamic model for semiconductor devices with application to a GaAs MESFET. The hydrodynamic model for semiconductor devices may be viewed as a second-order perturbation of a nonlinear hyperbolic system for $n$, the electron density, $\mathbf{p}$, the momentum density, and $w$, the energy density,

$$
\begin{aligned}
\partial_{t} n & +\operatorname{div}(n \mathbf{v})=0, \\
\partial_{t} \mathbf{p} & +\mathbf{v} \operatorname{div} \mathbf{p}+\mathbf{p} \cdot \nabla \mathbf{v}+\nabla(n k T) \\
& =-e n \mathbf{E}+\left(\partial_{t} \mathbf{p}\right)_{c}, \\
\partial_{t} w & +\operatorname{div}(\mathbf{v} w)+\operatorname{div}(\mathbf{v} n k T) \\
& =-e n \mathbf{v} \cdot \mathbf{E}+\left(\partial_{t} w\right)_{c}+\operatorname{div}(\kappa \nabla T),
\end{aligned}
$$

\footnotetext{
${ }^{1}$ Department of Mathematics and the Institute for Scientific Computation, Texas A\& M University, College Station, TX 77843.

${ }^{2}$ School of Mathematics, University of Minnesota, Minneapolis, Minnesota 55455.
}

where $k$. is Boltzmann's constant and the velocity $\mathbf{v}$ and the temperature $T$ are given by

$$
\begin{aligned}
\mathbf{p} & =m n \mathbf{v}, \\
w & =\frac{3}{2} n k T+\frac{1}{2} m n|\mathbf{v}|^{2},
\end{aligned}
$$

where $m$ is the effective electron mass. These equations are coupled with Poisson's equation defining the electric field $\mathbf{E}$ :

$$
\begin{aligned}
\mathbf{E} & =-\nabla \phi, \\
\operatorname{div}(\varepsilon \nabla \phi) & =-e\left(N_{D}-N_{A}-n\right),
\end{aligned}
$$

where $\varepsilon$ is the dielectric constant, and $N_{D}$ and $N_{A}$ are the densities of donors and acceptors, respectively. The constant $e(>0)$ is the electronic charge and $\kappa$ is the heat conduction coefficient. The "collision" terms are obtained by defining the momentum

\footnotetext{
${ }^{3}$ Department of Mathematics, Northwestern University, Evanston, IL 60208.

${ }^{4}$ Division of Applied Mathematics, Brown University, Providence, RI 02912.
} 
and energy relaxation times, $\tau_{\mathbf{p}}$ and $\tau_{w}$, following [1], [14], [16], as

$$
\begin{aligned}
\left(\partial_{t} \mathbf{p}\right)_{c} & =-\frac{\mathbf{p}}{\tau_{\mathbf{p}}}, \quad \tau_{\mathbf{p}}=m \frac{\mu_{n 0}}{e} \frac{T_{0}}{T}, \\
\left(\partial_{t} w\right)_{c} & =-\frac{w-\frac{3}{2} n k T_{0}}{\tau_{w}}, \\
\tau_{w} & =\frac{\tau_{\mathbf{p}}}{2}+\frac{3}{2} \frac{\mu_{n 0}}{e v_{s}^{2}} \frac{k T T_{0}}{T+T_{0}},
\end{aligned}
$$

where $T_{0}$ is the ambient temperature, $\mu_{n 0}=$ $\mu_{n 0}\left(T_{0}, N_{D}+N_{A}\right)$ is the low field electron mobility, and $v_{s}=v_{s}\left(T_{0}\right)$ is the saturation velocity. Finally, $\kappa$ is determined by the Wiedemann-Franz law [2]

$$
\kappa=\kappa_{0} \frac{\mu_{n 0}}{e} n k^{2} T\left(\frac{T}{T_{0}}\right)^{r} .
$$

In this paper, we take $r=-1$. We have selected a MESFET because of its acknowledged importance, particularly in microwave applications. It represents an application for which numerical methods are required to be robust over a wide parameter regime, although in this paper we restrict attention to ambient room temperature. We emphasize the importance of retention of the convective term, $\mathbf{p} \cdot \nabla \mathbf{v}$, in (1.1b), if a robust model is desired. This is the term which permits shocks in the hydrodynamic model when present. Such shocks were first identified in the $n^{+}-n-n^{+}$diode in [12] (see also [13]). The first study of the hydrodynamic model making use of shock capturing methods per se, which are effective even in the absence of shocks, was carried out in [10]. In [11], two independent numerical methods were coordinated in capturing shocks. Shocks are not reported in this paper, however.

To devise our numerical method, we first notice that the left-hand side of (1.1) defines a nonlinear hyperbolic operator (identical to that of the Euler equations of gas dynamics). As a consequence, to discretize (1.1) it is reasonable to use an efficient method originally devised for nonlinear conservation laws. We shall thus use the Runge-Kutta Discontinuous Galerkin (RKDG) method; see [4], [5], [6], [7], and [8]. This method is an explicit method which is fully parallelizable. It combines the advantages of the best finite difference methods for conservation laws (stability, high-order accuracy, element by element enforcement of conservation, sharp resolution of discontinuities), with those of finite element and volume methods (easy handling of complicated geometries, use of general triangulations for adaptiv- ity, and easy treatment of boundary conditions). Since the RKDG method is explicit, to advance in time we need a way to compute the right-hand side of (1.1) for a given $(n, \mathbf{p}, w)$. We notice that since only the electric field, and not the electric potential, appears on the right-hand side of (1.1), we should use a method that provides a direct approximation to the electric field $\mathbf{E}$. We can thus use any standard mixed finite element method to discretize the equations (1.2); we can discretize (1.2) with a mesh different from the one used by the RKDG method. To compute the divergence of the heat flux we use a simple $L^{2}$-projection method.

In this paper, we only use triangulations made of rectangles, since our main computational goal is to fairly compare our results with those of the Essentially Non-Oscillatory (ENO) finite difference method; see [18], [19], [20], [10], and [15]. The paper [15] contains the first two-dimensional simulations of the hydrodynamic model using finite difference shock-capturing methods. The approximation to $n$, each of the components of $\mathbf{p}$, and $w$, will be taken to be piecewise linear (or piecewise constant). In accordance with this choice, we use the RaviartThomas mixed method of lowest order [17]. We use the so-called Lagrange multipliers to be able to solve the mixed method by inverting a symmetric positive definite matrix.

We test our method on a MESFET device. Our numerical experiments show that (i) the results using a piecewise linear approximation for $n, \mathbf{p}$, and $w$ are by far superior to those obtained with a piecewise constant approximation, that (ii) the sharp gradients, boundary layers, and cusps of the solution are very well captured by the method (this is particularly clear in the case of the velocity field), and that (iii) our results are comparable to those obtained with the ENO finite difference method. These results indicate that our method is a very promising method for numerically solving the equations of the hydrodynamic model.

This paper is organized as follows. In $\$ 2$, we define our method in detail. In $\$ 3$, we present and discuss our numerical results. Finally, in $\$ 4$ we end with some concluding remarks.

\section{THE NUMERICAL METHOD}

\section{a. General Description}

To describe our numerical method, we first write the initial boundary value problem for 
$\mathbf{u}=\left(n, p_{x}, p_{y}, w\right)^{t}$ as follows:

$$
\begin{aligned}
\partial_{t} \mathbf{u}+\operatorname{div} \mathbf{F}(\mathbf{u}) & =\mathbf{R}(\mathbf{u}), & & \text { in }(0, T) \times \Omega, \\
\mathbf{u}(t=0) & =\mathbf{u}_{0}, & & \text { on } \Omega, \\
\mathbf{B u} & =\mathbf{g}, & & \text { on }(0, T) \times \partial \Omega,
\end{aligned}
$$

where the flux $F=\left(f_{x}, f_{y}\right)$ has the following components:

$$
\begin{aligned}
& \mathbf{f}_{x}(\mathbf{u})=v_{x} \mathbf{u}+\left(0, n k T, 0, v_{x} n k T\right)^{t} \\
& \mathbf{f}_{y}(\mathbf{u})=v_{y} \mathbf{u}+\left(0,0, n k T, v_{y} n k T\right)^{t},
\end{aligned}
$$

the right-hand side $\mathbf{R}$ is given by

$$
\begin{aligned}
\mathbf{R}(\mathbf{u}) & =\xi_{\mathbf{E}}(\mathbf{u})+\xi_{c}(\mathbf{u})+\xi_{\text {heat }}(\mathbf{u}), \\
\xi_{\mathbf{E}}(\mathbf{u}) & =\left(0,-e n E_{x},-e n E_{y},-e n \mathbf{v} \cdot \mathbf{E}\right)^{t}, \\
\xi_{c}(\mathbf{u}) & =\left(0,\left(\partial_{t} p_{x}\right)_{c},\left(\partial_{t} p_{y}\right)_{c},\left(\partial_{t} w\right)_{c}\right)^{t}, \\
\xi_{\text {heat }}(\mathbf{u}) & =(0,0,0, \operatorname{div}(\kappa \nabla T))^{t},
\end{aligned}
$$

and $\mathbf{B}$ a matrix-valued function. For the moment, we assume that we know how to obtain $\mathbf{R}(\mathbf{u})$ for a given $\mathbf{u}$.

An overview of the discretization of our equations is as follows. First, we triangulate our domain $\Omega$ with triangulations $\mathscr{T}_{h}$ made solely of rectangles $R$ such that the intersection of two distinct rectangles of the triangulation $\mathscr{T}_{h}$ is either an edge, a vertex, or void. Then, for each $t \in(0, T]$, we take each of the components of our approximate solution $\mathbf{u}_{h}(t)$ in the space

$$
V_{h}=\left\{p \in L^{\infty}(\Omega):\left.p\right|_{R} \text { is linear, } \forall R \in \mathscr{T}_{h}\right\} .
$$

We define each of the components of $\mathbf{u}_{0 h}$ to be the $\mathrm{L}^{2}$-projection of the corresponding component of $\mathbf{u}_{0}$ into $V_{h}$ and discretize the equation (2.1a) in space by using the Discontinuous Galerkin (DG) method which will be described in detail later. Since the functions of the space $V_{h}$ are discontinuous, the mass matrix of the DG method is block-diagonal and hence easily invertible by hand. Thus, the resulting discrete equations can be rewritten as the following ODE initial value problem:

$$
\begin{aligned}
& \frac{d \mathbf{u}_{h}}{d t}=\mathbf{L}_{h}\left(\mathbf{u}_{h}, \mathbf{g}\right)+\mathbf{R}_{h}\left(\mathbf{u}_{h}\right), \quad t \in(0, T], \\
& \mathbf{u}_{h}(t=0)=\mathbf{u}_{0 h},
\end{aligned}
$$

where $\mathbf{L}_{h}$ is the approximation of $-\operatorname{div} \mathbf{F}$. The exact solution of the above initial value problem gives an approximation which is formally second-order accurate in space; see [7]. Accordingly, a second-order accurate in time Runge-Kutta method must be used to discretize our ODE; see [7] and [18]. Finally, a local projection $\Lambda \Pi_{h}$ is applied to the intermediate values of the Runge-Kutta discretization in order to enforce nonlinear stability.

The resulting formally second-order accurate scheme reads as follows:

$$
\text { Set } \mathbf{u}_{h}^{0}=\Lambda \Pi_{h}\left(\mathbf{u}_{0 h}\right)
$$

For $n=0, \ldots, n_{T}-1$, compute $\mathbf{u}_{h}^{n+1}$ as follows:

(i) set $\mathbf{u}_{h}^{[0]}=\mathbf{u}_{h}^{n}$;

(ii) compute $\mathbf{u}_{h}^{[1]}$ and $\mathbf{u}_{h}^{[2]}$ as follows:

$$
\begin{aligned}
\mathbf{u}_{h}^{[1]}= & \Lambda \Pi_{h}\left(\mathbf{u}_{h}^{n}+\Delta t^{n} \mathbf{L}_{h}\left(\mathbf{u}_{h}^{[0]}, \mathbf{g}\left(t^{n}\right)\right)\right. \\
& \left.+\Delta t^{n} \mathbf{R}_{h}\left(\mathbf{u}_{h}^{[0]}\right)\right), \\
\mathbf{w}_{h}= & \mathbf{u}_{h}^{[1]}+\Delta t^{n} \mathbf{L}_{h}\left(\mathbf{u}_{h}^{[1]}, \mathbf{g}_{h}\left(t^{n+1}\right)\right) \\
& \left.+\Delta t^{n} \mathbf{R}_{h}\left(\mathbf{u}_{h}^{[1]}\right)\right), \\
\mathbf{u}_{h}^{[2]}= & \Lambda \Pi_{h}\left(\left(\mathbf{u}_{h}^{n}+\mathbf{w}_{h}\right) / 2\right) ;
\end{aligned}
$$

(iii) Set $\mathbf{u}_{h}^{n+1}=\mathbf{u}_{h}^{[2]}$.

In what follows, we describe in detail the approximation of the divergence $-\mathbf{L}_{h}$, the local projection $\Lambda \Pi_{h}$, and the right-hand side $\mathbf{R}_{h}$.

\section{b. The Discontinuous Galerkin Method}

The general definition of the DG method in the case of a scalar $\mathbf{u}$ can be found in [7]. To define the method in our case, we simply have to apply the procedure for the scalar case component by component. For the sake of completeness, we display such a procedure.

Let us denote by $\mathbf{u}^{(k)}$ the $k$-th component of the vector $\mathbf{u}$. Consider the equation for the $k$-th component of the system (2.1a), multiply it by $v_{h} \in V_{h}$, 
integrate over each $R \in \mathscr{T}_{h}$, replace the exact solution $\mathbf{u}$ by its approximation $\mathbf{u}_{h}$, and formally integrate by parts to obtain

$$
\begin{aligned}
& \frac{d}{d t} \int_{R} \mathbf{u}_{h}^{\{k\}}(t, x, y) v_{h}(x, y) d x d y \\
& \quad+\sum_{e \in \partial R} \int_{e} \mathbf{F}^{\{k\}}\left(\mathbf{u}_{h}(t, x, y)\right) \\
& \quad \cdot \mathbf{n}_{e, R} v_{h}(x, y) d \Gamma(x, y) \\
& \quad-\int_{R} \mathbf{F}^{\{k\}}\left(\mathbf{u}_{h}(t, x, y)\right) \cdot \nabla v_{h}(x, y) d x d y \\
& =\int_{R} \mathbf{R}^{\{k\}}\left(\mathbf{u}_{h}(t, x, y)\right) v_{h}(x, y) d x d y, \forall v_{h} \in V_{h},
\end{aligned}
$$

where $\mathbf{n}_{e, R}$ is the outward unit normal to the edge $e$. Notice that $\mathbf{F} \cdot \mathbf{n}=\mathbf{f}_{x} n_{x}+\mathbf{f}_{y} n_{y}$ is a four-dimensional vector whose $k$-th component is $\mathbf{F}^{(k)} \cdot \mathbf{n}=$ $\mathbf{f}_{x}^{(k)} n_{x}+\mathbf{f}_{y}^{(k)} n_{y}$. Notice also that $\mathbf{F}\left(\mathbf{u}_{h}(t, x, y)\right) \cdot \mathbf{n}_{e, R}$ does not have a precise meaning, since $\mathbf{u}_{h}$ is discontinuous at $(x, y) \in e \in \partial R$. Thus, we replace $\mathbf{F}\left(\mathbf{u}_{h}(t, x, y)\right) \cdot \mathbf{n}_{e, R}$ by a suitably chosen numerical flux $\mathbf{h}_{e, R}$, which depends on the two values of $\mathbf{u}_{h}$ on the edge $e, \mathbf{u}_{h}\left((x, y)^{i n t(R)}\right)$ and $\mathbf{u}_{h}\left((x, y)^{e x t(R)}\right)$, defined as follows:

$$
\begin{gathered}
\mathbf{u}_{h}\left(t,(x, y)^{i n t(R)}\right) \\
=\lim _{\left(x^{\prime}, y^{\prime}\right) \rightarrow(x, y),\left(x^{\prime}, y^{\prime}\right) \in(R)^{0}} \mathbf{u}_{h}\left(t, x^{\prime}, y^{\prime}\right), \\
\mathbf{u}_{h}\left(t,(x, y)^{e x t(R)}\right) \\
=\lim _{\left(x^{\prime}, y^{\prime}\right) \rightarrow(x, y),\left(x^{\prime}, y^{\prime}\right) \in(R)^{c}} \mathbf{u}_{h}\left(t, x^{\prime}, y^{\prime}\right), \\
\mathbf{B u}_{h}(t,(x, y) \notin \partial \Omega, \\
\left.(x, y)^{\operatorname{ext}(R)}\right)=\mathbf{g}_{h}(t, x, y),(x, y) \in \partial \Omega .
\end{gathered}
$$

The choice of this numerical flux is crucial since it is through the use of the numerical flux that the upwinding (or the artificial viscosity) which renders the method stable (without destroying its high-order accuracy) is introduced. In this paper, we choose the so-called local Lax-Friedrichs flux which will be described in the next section.
Finally, we replace the integrals above by quadrature rules:

$$
\begin{aligned}
& \int_{e}^{\mathbf{h}_{e, R}^{\{k\}}(t, x, y) v_{h}(x, y) d \Gamma(x, y)} \\
& \simeq \sum_{l=1}^{L} \omega_{l} \mathbf{h}_{e, R}^{\{k\}}\left(t,(x, y)_{e l}\right) v_{h}\left((x, y)_{e l}\right)|e|, \\
& \int_{R} \mathbf{F}^{(k)}\left(\mathbf{u}_{h}(t, x, y)\right) \cdot \nabla v_{h}(x, y) d x d y \\
& \simeq \sum_{l=1}^{L L} \hat{\boldsymbol{\omega}}_{l} \mathbf{F}^{\{k\}}\left(\mathbf{u}_{h}\left(t,(x, y)_{R l}\right)\right) \\
& \cdot \nabla v_{h}\left((x, y)_{R l}\right)|R|,
\end{aligned}
$$

where $\quad \mathbf{h}_{e, R}(t, x, y)=\mathbf{h}_{e, R}\left(\mathbf{u}_{h}\left(t,(x, y)^{i n t(R)}\right)\right.$, $\mathbf{u}_{\mathrm{h}}\left(t,(x, y)^{e x t(R)}\right)$ and $\omega_{l}$ and $\hat{\omega}_{l}$ are integration weights. Following [7, Proposition 2.1], we take the two-point Gauss quadrature formula for the edges and the four-point Gauss quadrature formula for the rectangles.

In this way, the weak formulation for approximating the solution to (2.1) is as follows. For each $k=1,2,3,4$, we have, $\forall v_{h} \in V_{h}$ and $\forall R \in \mathscr{T}_{h}$,

$$
\begin{aligned}
& \frac{d}{d t} \int_{R} \mathbf{u}_{h}^{k\}}(t, x, y) v_{h}(x, y) d x d y \\
& +\sum_{e \in \partial R} \sum_{l=1}^{L} \omega_{l} \mathbf{h}_{e, R}^{\{k\}}\left(t,(x, y)_{e l}\right) \\
& \quad \times v_{h}\left((x, y)_{e l}\right)|e| \\
& -\sum_{l=1}^{L L} \hat{\omega}_{l} \mathbf{F}^{(k)}\left(\mathbf{u}_{h}\left(t,(x, y)_{R l}\right)\right) \\
& \quad \cdot \nabla v_{h}\left((x, y)_{R l}\right)|R| \\
& =\sum_{l=1}^{L L} \hat{\omega}_{l} \mathbf{R}^{\{k\}}\left(\mathbf{u}_{h}\left(t,(x, y)_{R l}\right)\right) v_{h}\left((x, y)_{R l}\right)|R| .
\end{aligned}
$$

The above weak formulation defines the operators $\mathbf{L}_{h}$ and $\mathbf{R}_{h}$.

\section{c. The Degrees of Freedom for $\mathbf{u}_{h}$}

Notice that the above weak formulation is completely independent of the way in which we choose 
to express our approximate solution. We have chosen to express our approximate solution $\mathbf{u}_{h}$ as follows. For $(x, y)$ in the rectangle

$$
\begin{aligned}
R= & \left(x_{i}-\Delta x_{i} / 2, x_{i}+\Delta x_{i} / 2\right) \\
& \times\left(y_{j}-\Delta y_{j} / 2, y_{j}+\Delta y_{j} / 2\right)
\end{aligned}
$$

we write

$$
\mathbf{u}_{h}(x, y)=\overline{\mathbf{u}}_{i j}+\left(2 \frac{x-x_{i}}{\Delta x_{i}}\right) \tilde{\mathbf{u}}_{x i j}+\left(2 \frac{y-y_{j}}{\Delta y_{j}}\right) \tilde{\mathbf{u}}_{y i j}
$$

that is, we choose as degrees of freedom of $\mathbf{u}_{h}$, its mean on $R, \overline{\mathbf{u}}_{i j}$, its variation in the $x$-direction, $\tilde{\mathbf{u}}_{x i j}$, and its variation in the $y$-direction, $\tilde{\mathbf{u}}_{y i j}$.

This choice of degrees of freedom renders the mass matrix of our weak formulation a $3 \times 3$ diagonal matrix. More importantly, this choice greatly facilitates the evaluation of the numerical flux $\mathbf{h}_{e, R}$ and the computation of the nonlinear projection $\Lambda \Pi_{h}$ which then becomes equivalent to two onedimensional nonlinear projections; see [6].

In order not to have to make a distinction between boundary and interior edges in the evaluation of the numerical flux $\mathbf{h}_{e, R}$ and in the computation of the nonlinear projection $\Lambda \Pi_{h}$, we express the boundary values as follows. Suppose that $(x, y)$ lies on the boundary edge $\left\{x_{i}+\Delta x_{i} / 2\right\} \times\left(y_{j}-\right.$ $\left.\Delta y_{j} / 2, y_{j}+\Delta y_{j} / 2\right)$; then we write the boundary values of $\mathbf{u}_{h}$ as

$$
\mathbf{u}_{h}(x, y)=\overline{\mathbf{u}}_{i+1, j}+\left(2 \frac{y-y_{j}}{\Delta y_{j}}\right) \tilde{\mathbf{u}}_{y i+1, j} .
$$

We use a similar representation of the boundary values of $\mathbf{u}$ on the boundary edge $\left(x_{i}-\Delta x_{i} / 2, x_{i}+\right.$ $\left.\Delta x_{i} / 2\right) \times\left\{y_{j}+\Delta y_{j} / 2\right\}$.

\section{d. The Local Projection $\Lambda \Pi_{h}$}

The local projection is devised to prevent the appearance of spurious oscillations in the approximate solution. The local averages, $\overline{\mathbf{u}}_{i j}$, are unchanged to preserve the conservativity of the method, but the local variation in the $x$-direction, $\tilde{\mathbf{u}}_{x i j}$, and the local variation in the $y$-direction, $\tilde{\mathbf{u}}_{y i j}$, must be controlled to avoid the unwanted oscillations. We can obtain some control on the oscillations along the $x$-direction with the following component by component algorithm. For the $k$-th component of the approximate solution we set

$$
\begin{aligned}
&\left(\tilde{\mathbf{u}}_{x i j}^{(m o d)}\right)^{\{k\}}=\operatorname{minmod}_{i}(\left(\tilde{\mathbf{u}}_{x i j}\right)^{\{k\}}, \\
&\left.\left(\Delta_{x+} \overline{\mathbf{u}}_{i j}\right)^{\{k\}},\left(\Delta_{x-} \overline{\mathbf{u}}_{i-1, j}\right)^{\{k\}}\right),
\end{aligned}
$$

where $\Delta_{x+}$ and $\Delta_{x-}$ denote the standard forward and backward finite difference operators in the $x$ direction and

$$
\begin{aligned}
& \operatorname{minmod}_{i}\left(a, b_{1}, \ldots, b_{n}\right) \\
& = \begin{cases}a, & \text { if }|a| \leq M \cdot\left(\Delta x_{i}\right)^{2}, \\
(\operatorname{sign} a) \min _{1 \leq l \leq n}\left\{|a|,\left|b_{l}\right|\right\}, & \text { if sign } a=\operatorname{sign} b_{l}, \\
0, & 1 \leq l \leq n, \\
0, & \text { otherwise, }\end{cases}
\end{aligned}
$$

where $n$ is an integer and $M$ is an upper bound of the second-order $x$-derivative of each of the components of $\mathbf{u}$; see [4]. The choice of $M$ will be discussed in $\S 4$. To try to control the oscillation along the $y$-direction, a similar algorithm may be used. However, although this algorithm is computationally efficient, it does not take into account the physically relevant directions along which the information travels. Taking these characteristic directions into account results in a better control of the oscillations and in a higher quality of the approximation.

Let us show how to do this to define $\tilde{\mathbf{u}}_{x i j}^{\text {(mod) }}$. First, we compute the Jacobians

$$
J_{i j}=\left(\frac{\partial \mathbf{F}}{\partial \mathbf{u}}\right) \cdot(1,0)\left(\tilde{\mathbf{u}}_{i j}\right),
$$

and obtain their eigenvalues and their left and right eigenvectors $\lambda_{i j}^{(p)}, \mathbf{l}_{i j}^{(p)}$, and $\mathbf{r}_{i j}^{(p)}, p=1, \ldots, 4$, respectively. The eigenvalues are normalized so that $\mathbf{l}_{i j}^{(p)}$. $\mathbf{r}_{i j}^{(q)}=\delta_{p q}$. Then, we project $\tilde{\mathbf{u}}_{x i j}, \Delta_{x+} \overline{\mathbf{u}}_{i j}$, and $\Delta_{x-} \overline{\mathbf{u}}_{i j}$ into the eigenspace of $J_{i j}$ :

$$
\begin{aligned}
& a^{(p)}=\mathbf{l}_{i j}^{(p)} \cdot \tilde{\mathbf{u}}_{x i j}, \quad p=1, \ldots, 4, \\
& b^{(p)}=\mathbf{l}_{i j}^{(p)} \cdot \Delta_{x+} \overline{\mathbf{u}}_{i J}, \quad p=1, \ldots, 4, \\
& c^{(p)}=\mathbf{l}_{i j}^{(p)} \cdot \Delta_{x-} \overline{\mathbf{u}}_{i j}, \quad p=1, \ldots, 4,
\end{aligned}
$$

and perform the projection (or slope limiting) in each characteristic field

$$
\tilde{u}_{x i j}^{(p)}=\operatorname{minmod}_{i}\left(a^{(p)}, b^{(p)}, c^{(p)}\right) .
$$

Next, we project them back to the component space 
to obtain

$$
\tilde{\mathbf{u}}_{x i j}^{(m o d)}=\sum_{p=1}^{4} \tilde{u}_{x i j}^{(p)} \mathbf{r}_{i j}^{(p)}
$$

This completes the projection in the $x$-direction; see [6]. A similar and totally independent procedure is applied in the $y$-direction.

\section{e. The Numerical Flux $h_{e, R}$}

The numerical flux we use is the so-called (componentwise) local Lax-Friedrichs flux. We define it as follows. Suppose that $R$ is the rectangle $\left(x_{i}-\right.$ $\left.\Delta x_{i} / 2, \quad x_{i}+\Delta x_{i} / 2\right) \times\left(y_{j}-\Delta y_{j} / 2, \quad y_{j}+\Delta y_{j} / 2\right)$ and suppose that the quadrature point $(x, y)_{e l}$ lies on the edge $\left\{x_{i}+\Delta x_{i} / 2\right\} \times\left(y_{j}-\Delta y_{j} / 2, y_{j}+\right.$ $\left.\Delta y_{j} / 2\right)$. Then,

$$
\begin{array}{r}
\mathbf{h}_{e, R}\left((x, y)_{e l}\right)=\frac{1}{2}\left\{\left(\mathbf{F}\left(\mathbf{u}_{h}\left((x, y)_{e l}^{i n t(R)}\right)\right) \cdot \mathbf{n}_{e, R}\right.\right. \\
+\mathbf{F}\left(\mathbf{u}_{h}\left((x, y)_{e l}^{\operatorname{ext}(R)}\right) \cdot \mathbf{n}_{e, R}\right) \\
\left.-\alpha_{e}\left(\mathbf{u}_{h}\left((x, y)_{e l}^{\operatorname{ext}(R)}\right)-\mathbf{u}_{h}\left((x, y)_{e l}^{i n t(R)}\right)\right)\right\}
\end{array}
$$

where

$$
\alpha_{e l}=\max \left\{\lambda\left(\overline{\mathbf{u}}_{i j}\right), \lambda\left(\overline{\mathbf{u}}_{i+1, j}\right)\right\}
$$

and

$$
\lambda\left(\left(n, p_{x}, p_{y}, w\right)^{t}\right)=\sqrt{\frac{5}{3} k T / m}+|\mathbf{v}|,
$$

is an upper bound for the eigenvalues of the Jacobian of $\mathbf{F} \cdot \nu$ evaluated at $\left(n, p_{x}, p_{y}, w\right)^{t}$ for all unit vectors $\nu$. Similar expressions hold for quadrature points in the remaining of the boundary of $R$.

A characteristicwisely evaluated local LaxFriedrichs flux can also be used. However, our experience is that the componentwisely evaluated local Lax-Friedrichs flux produces as good results as this more costly flux.

\section{f. The Right-Hand Side $\mathbf{R}\left(\mathrm{u}_{h}\right)$}

In this section we show how to evaluate the function $\mathbf{R}_{h}\left(\mathbf{u}_{h}\right)=\xi_{\mathbf{E}}\left(\mathbf{u}_{h}\right)+\xi_{c}\left(\mathbf{u}_{h}\right)+\xi_{\text {heat }}\left(\mathbf{u}_{h}\right)$ for a given $\mathbf{u}_{h}$. To evaluate $\xi_{c}\left(u_{h}\right)$, we simply use the equations (1.4) and (1.2).
To evaluate $\xi_{\mathbf{E}}\left(\mathbf{u}_{h}\right)$, we need a numerical method to obtain an approximation to the electric field $\mathbf{E}, \mathbf{E}_{h}$. The equations defining the electric field are the equations (1.3) and some boundary conditions we write as follows:

$$
\begin{array}{ll}
\operatorname{div} \mathbf{E}=\frac{e}{\varepsilon}\left(N_{D}-N_{A}-n\right) & \text { in } \Omega, \\
\mathbf{E}=-\nabla \phi & \text { in } \Omega, \\
\phi=\phi_{D} & \text { on } \partial \Omega_{D}, \\
\mathbf{E} \cdot \mathbf{n}=0 & \text { on } \partial \Omega_{N},
\end{array}
$$

where $\partial \Omega=\partial \Omega_{D} \cup \partial \Omega_{N}$ and $\partial \Omega_{D} \cap \partial \Omega_{N}=\varnothing$. We discretize these equations with the lowest-order Raviart-Thomas mixed method which defines the approximation $\left(\mathbf{E}_{h}, \phi_{h}\right) \in \mathbf{U}_{h} \times W_{h}$ as the solution of the following weak formulation:

$$
\begin{aligned}
& \left(\operatorname{div} \mathbf{E}_{h}, w\right)=\left(\frac{e}{\varepsilon}\left(N_{D}-N_{A}-n_{h}\right), w\right), \forall w \in W_{h}, \\
& \left(\mathbf{E}_{h}, \mathbf{v}\right)-\left(\phi_{h}, \operatorname{div} \mathbf{v}\right)=-\left\langle\phi_{D}, \mathbf{v} \cdot \mathbf{n}\right\rangle_{\partial \Omega_{D}}, \forall \mathbf{v} \in \mathbf{U}_{h},
\end{aligned}
$$

where $n_{h}$ is the approximate density yielded by the RKDG method, and

$$
\begin{aligned}
& U_{h}=\left\{\mathbf{v} \in \mathbf{H}(\operatorname{div} ; \Omega):\left.\mathbf{v}\right|_{R}\right. \\
&=\left(a_{R}^{1}+a_{R}^{2} x, a_{R}^{3}+a_{R}^{4} y\right), a_{R}^{i} \in \mathbb{R}, \\
&\left.\forall R \in \mathscr{T}_{h} ;\left.\mathbf{v} \cdot \mathbf{n}\right|_{\partial \Omega_{N}}=0\right\},
\end{aligned}
$$

$W_{h}=\left\{w \in L^{2}(\Omega):\left.w\right|_{R}\right.$ is a constant, $\left.\forall R \in \mathscr{T}_{h}\right\}$.

It can be shown that the above system has a unique solution in $\mathbf{U}_{h} \times W_{h}$ [9], [17]. As pointed out before, we use the Lagrange multipliers (see for example [3] and the references therein) which render the matrix of the resulting method a symmetric positive definite matrix. We invert it by using the conjugate gradient method with incomplete Choleski factorization as preconditioner.

To evaluate $\xi_{\text {heat }}\left(\mathbf{u}_{h}\right)$ we also use the RaviartThomas spaces, although in a very different way. We proceed as follows. First, we rewrite (2.2d) as follows:

$$
\xi_{\text {heat }}(\mathbf{u})=(0,0,0, \operatorname{div}(\mathbf{q}))^{t}
$$


where $\mathbf{q}$ is defined by

$$
\begin{array}{ll}
\mathbf{q}=\kappa \nabla T, & \text { in } \Omega, \\
T=T_{D}, & \text { on } \partial \Omega_{D T}, \\
\mathbf{q} \cdot \mathbf{n}=0, & \text { on } \partial \Omega_{N T},
\end{array}
$$

where $\partial \Omega=\partial \Omega_{D T} \cup \partial \Omega_{N T}$ and $\partial \Omega_{D T} \cap \partial \Omega_{N T}=$ $\varnothing$. Then we define the approximation $\mathbf{q}_{h} \in \mathbf{Q}_{h}$ as the solution of the following weak formulation:

$$
\begin{aligned}
\left(\kappa_{h}^{-1} \mathbf{q}_{h}, \mathbf{v}\right)= & -\left(T_{h}, \operatorname{div} \mathbf{v}\right) \\
& +\left\langle T_{D}, \mathbf{v} \cdot \mathbf{n}\right\rangle_{\partial \Omega_{D T}}, \forall \mathbf{v} \in \mathbf{Q}_{h},
\end{aligned}
$$

where $T_{h}$ and $\kappa_{h}$ are given by (1.2b) and (1.5), respectively, and

$$
\begin{aligned}
& \mathbf{Q}_{h}=\left\{\mathbf{v} \in \mathbf{H}(\operatorname{div} ; \Omega):\left.\mathbf{v}\right|_{R}\right. \\
&=\left(a_{R}^{1}+a_{R}^{2} x, a_{R}^{3}+a_{R}^{4} y\right), a_{R}^{i} \in \mathbb{R}, \\
&\left.\forall R \in \mathscr{T}_{h} ;\left.\mathbf{v} \cdot \mathbf{n}\right|_{\partial \Omega_{N T}}=0\right\} .
\end{aligned}
$$

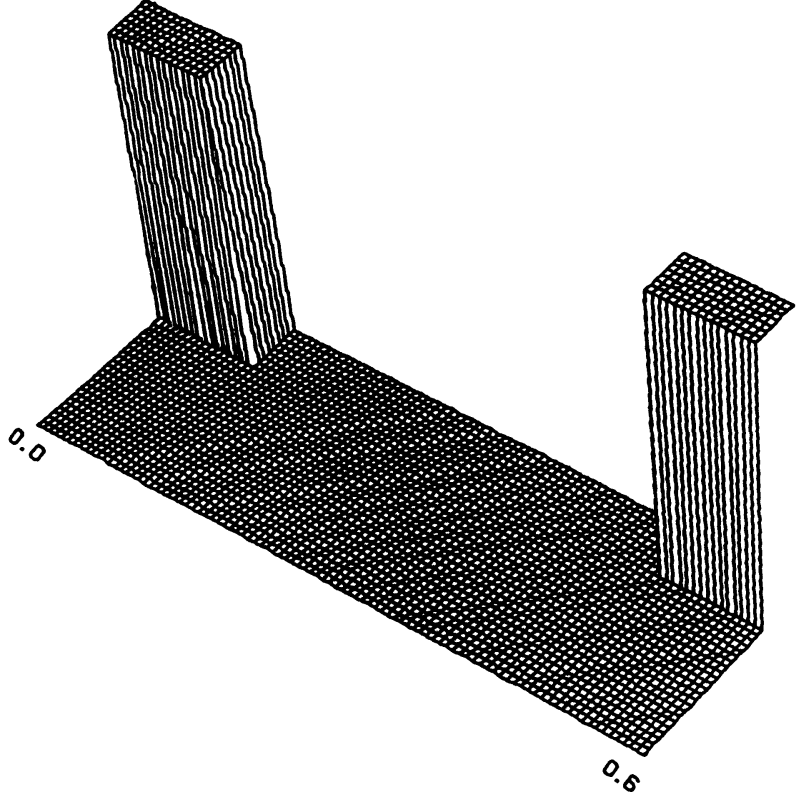

FIGURE 2 The doping profile $N_{D}$.

The doping $N_{D}$ is defined by
To solve for $\mathbf{q}_{h}$, we simply lump the mass matrix thus obtaining a diagonal system.

\section{NUMERICAL RESULTS}

A two dimensional MESFET device $\Omega=(0,0.6 \mu \mathrm{m})$ $\times(0,0.2 \mu \mathrm{m})$ is simulated, using the hydrodynamic model (1.1), (1.2), (1.3), (1.4), and (1.5). The source is the segment $(0,0.1 \mu \mathrm{m}) \times\{y=0.2 \mu \mathrm{m}\}$, the drain is the segment $(0.5 \mu \mathrm{m}, 0.6 \mu \mathrm{m}) \times\{y=0.2 \mu \mathrm{m}\}$, and the gate is the segment $(0.2 \mu m, 0.4 \mu m) \times\{y=$ $0.2 \mu m\}$; see Fig. 1 .

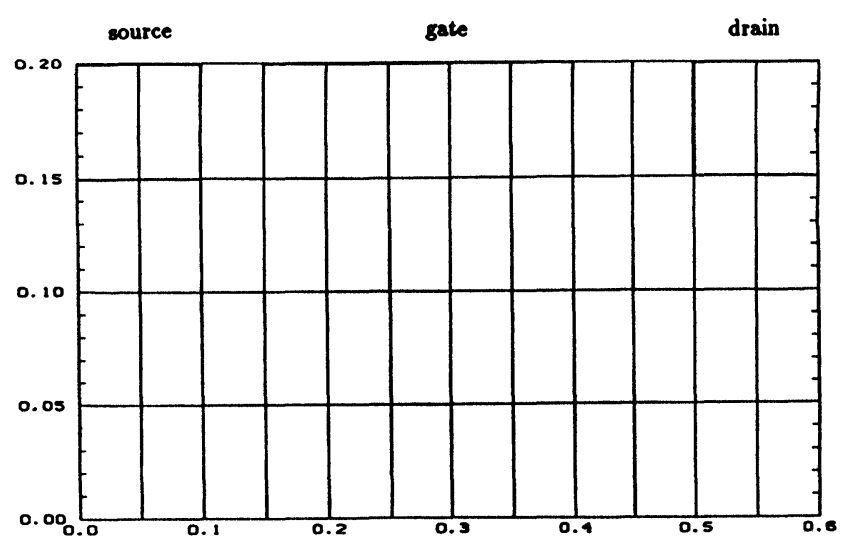

FIGURE 1 The MESFET semiconductor devices.
$N_{D}=\left\{\begin{array}{cc}3 \times 10^{17} \mathrm{~cm}^{-3}, & (x, y) \in[0,0.1] \\ & \times[0.15,0.2] \cup[0.5,0.6] \\ & \times[0.15,0.2] \\ 1 \times 10^{17} \mathrm{~cm}^{-3}, & \text { elsewhere }\end{array}\right.$

and $N_{A}=0$ in the whole domain $\Omega$; see Fig. 2 .

The initial conditions are chosen as $n=N_{D}$ for the density, $T=T_{0}=300^{\circ} \mathrm{K}$ for the temperature, and $v_{x}=v_{y}=0$ for the velocity. The initial condition for the potential is $\phi_{0}^{*}$, where $\phi_{0}^{*}=\phi_{0}-.232$, and

$$
\phi_{0}=k T_{0} \ln \left(\frac{N_{D}}{n_{i}}\right) / e
$$

with $k=0.138 \times 10^{-4}, e=0.1602$, and $n_{i}=$ 0.000018 (for GaAs). We are employing a translation constant 0.232 here for convenience in our simulations. The boundary condition is defined as follows:

(i) At the source: $\phi=\phi_{0}^{*}$ for the potential, $n=3 \times 10^{17} \mathrm{~cm}^{-3}$ for the electron density, $T=300^{\circ} \mathrm{K}$ for the temperature, $v_{x}=0 \mu \mathrm{m} / \mathrm{ps}$ for the horizontal velocity, and the homogeneous Neumann boundary condition for the vertical velocity $v_{y}$; 
(ii) At the drain: $\phi=\phi_{0}^{*}+2$ for the potential, $n=3 \times 10^{17} \mathrm{~cm}^{-3}$ for the electron density, $T=300^{\circ} \mathrm{K}$ for the temperature, $v_{x}=0 \mu \mathrm{m} / \mathrm{ps}$ for the horizontal velocity, and the homogeneous Neumann boundary condition for the vertical velocity $v_{y}$

(iii) At the gate: $\phi=\phi_{0}^{*}-0.8$ for the potential, $n=3.9 \times 10^{5} \mathrm{~cm}^{-3}$ for the electron density, $T=300^{\circ} \mathrm{K}$ for the temperature, $v_{x}=0 \mu \mathrm{m} / \mathrm{ps}$ for the horizontal velocity, and the homogeneous Neumann boundary condition for the vertical velocity $v_{y}$;

(iv) At all other parts of the boundary: all variables are subjected to homogeneous Neumann boundary conditions.

We simulate homogeneous Neumann boundary conditions for each of the components of $\mathbf{u}_{h}$ as follows. Suppose that the edge $\left\{x_{i}+\Delta x_{i} / 2\right\} \times$ $\left(y_{j}-\Delta y_{j} / 2, y_{j}+\Delta y_{j} / 2\right)$ lies on a Neumann boundary for, say, $\left(\mathbf{u}_{h}\right)^{\{k\}}$. Then we define the degrees of

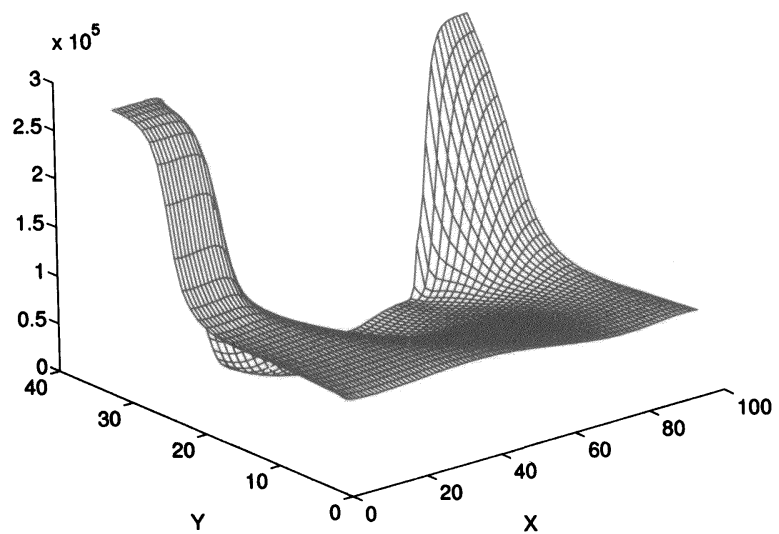

FIGURE 3A The density $n_{h}$ obtained with the $96 \times 32$ mesh.

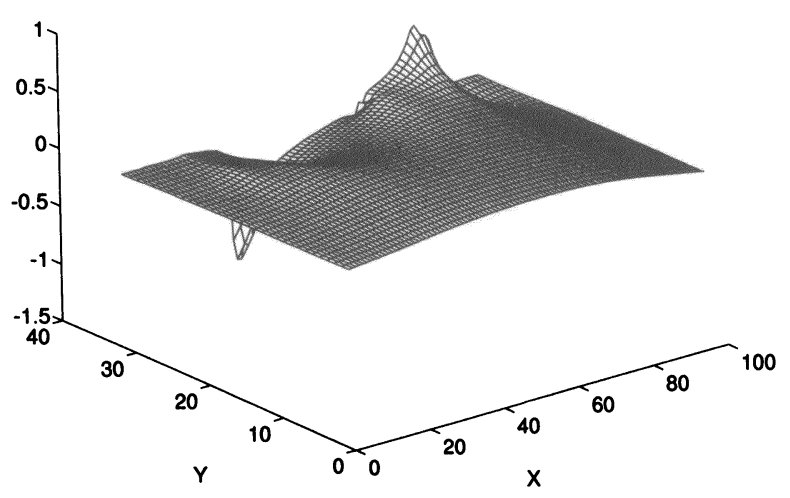

FIGURE 3B The $x$-component of the velocity $v_{x h}$ obtained with the $96 \times 32$ mesh. freedom of $\left(\mathbf{u}_{h}\right)^{\{k\}}$ at the boundary as follows:

$$
\left(\overline{\mathbf{u}}_{i+1, j}\right)^{\{k\}}=\left(\overline{\mathbf{u}}_{i, j}\right)^{\{k\}}, \quad\left(\tilde{\mathbf{u}}_{y i+1, j}\right)^{\{k\}}=\left(\tilde{\mathbf{u}}_{y i, j}\right)^{\{k\}} .
$$

Similar expressions are used on the other edges on the Neumann boundary. In all our simulations we chose $M=200$. We have also run the simulations with $M=2000$ without noticing any major difference. Uniform space meshes of $96 \times 32$ and $192 \times$ 64 are employed for the simulations in which the method is run until the steady state is reached. The use of general triangulations and of acceleration methods for steady state computations are the subject of future work. In this paper, we are mainly interested in comparing the results of our method with those given by the third-order accurate ENO shock-capturing algorithm with Lax-Friedrichs building blocks [18], [19]. This method is combined with a standard central difference treatment of the heat conduction term and the Poisson equation, which is solved by the SOR method; see [15] for more details.

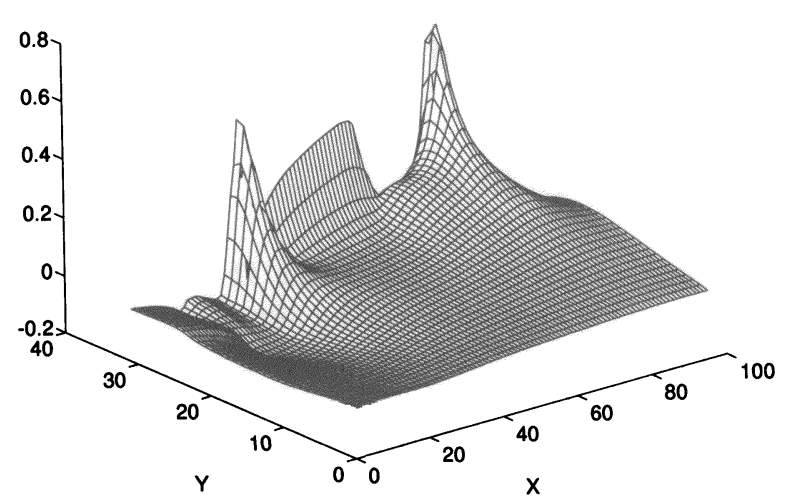

FIGURE 3C The $y$-component of the velocity $v_{y h}$ obtained with the $96 \times 32$ mesh.

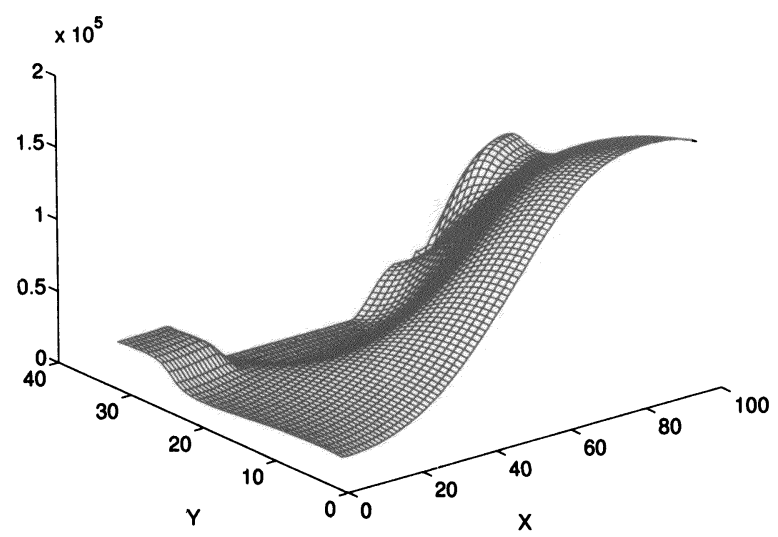

FIGURE 3D The energy $w_{h}$ obtained with the $96 \times 32$ mesh. 
To give an idea of how the proposed method works, we display the results of our method on the $96 \times 32$ mesh in Figs. 3. Notice the sharp transition of the electron density $n$ near the junctions. Also, notice the boundary layer for $n$ at the drain, but not at the source. This is reasonable since the drain is an outflow boundary and the source is an inflow

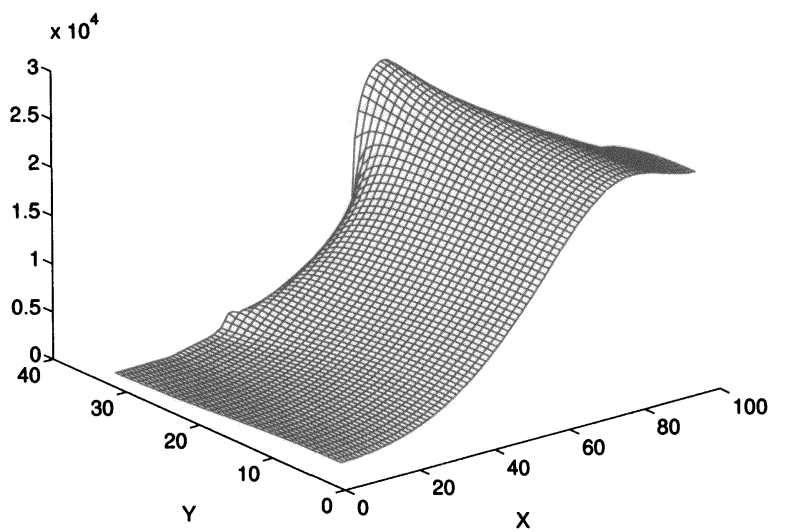

FIGURE 3E The temperature $T_{h}$ obtained with the $96 \times 32$ mesh.

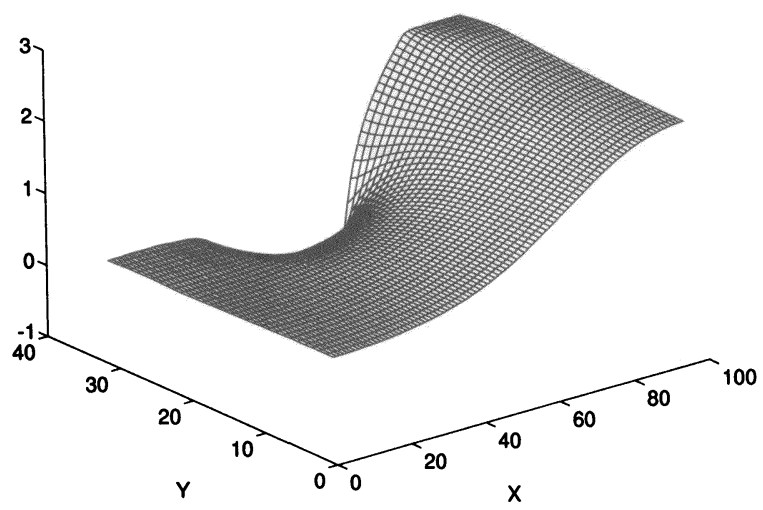

FIGURE 3F The electric potential $\phi_{h}$ obtained with the $96 \times$ 32 mesh.

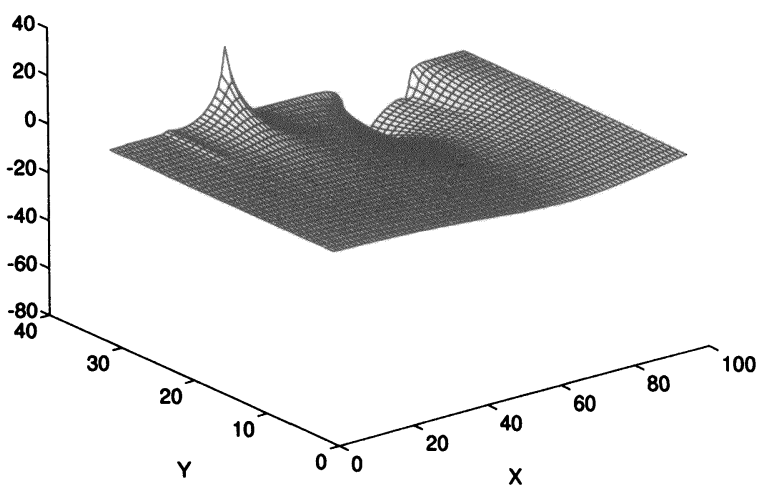

FIGURE 3G The $x$-component of the electric field $\mathbf{E}_{x h}$ obtained with the $96 \times 32$ mesh. boundary. A rapid drop of $n$ at the depletion region occurs near the gate. The normal velocity component at the gate appears to be negligible, while the horizontal component shows evidence of strong carrier movement toward the source beneath the left gate area, and strong movement toward the drain immediately to the left of the drain junction. Notice

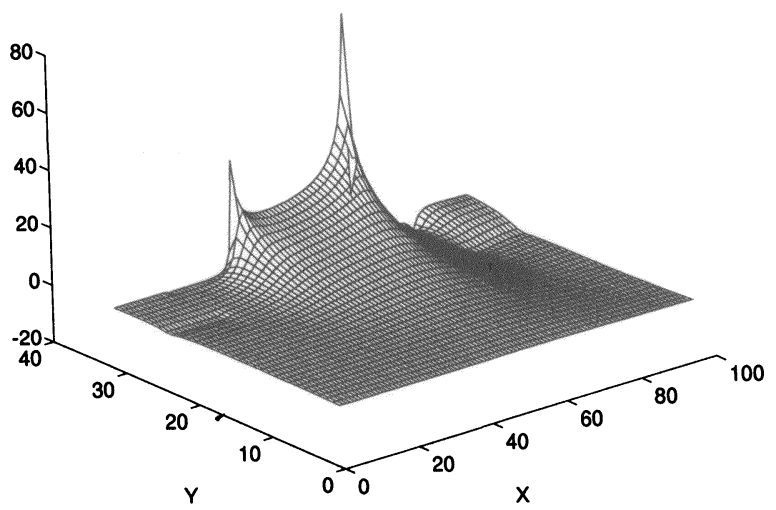

FIGURE 3H The $y$-component of the electric field $\mathbf{E}_{y h}$ obtained with the $96 \times 32$ mesh.

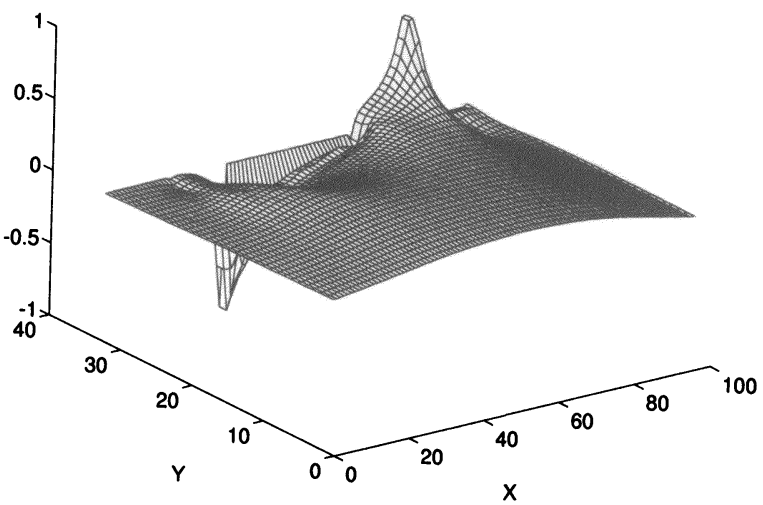

FIGURE $4 \mathrm{~A}$ The $v_{x h}$ for the $96 \times 32$ mesh: the $\mathrm{P}^{1}-\mathrm{RKGD}$ method.

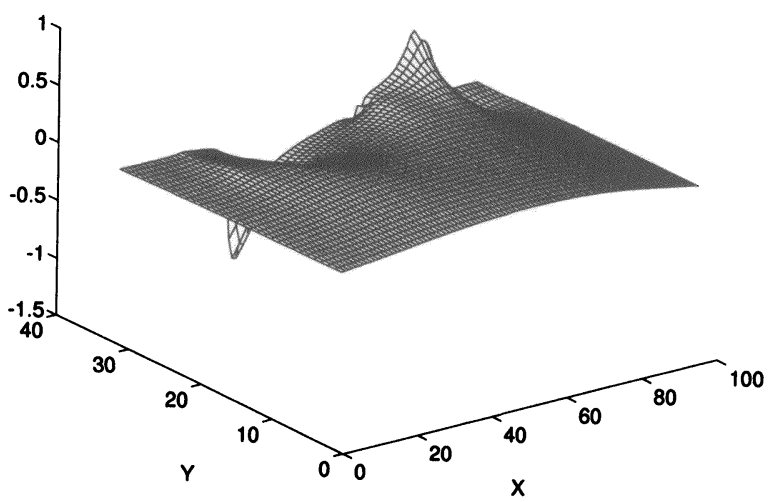

FIGURE 4B The $v_{x h}$ for the $96 \times 32$ mesh: the ENO method. 


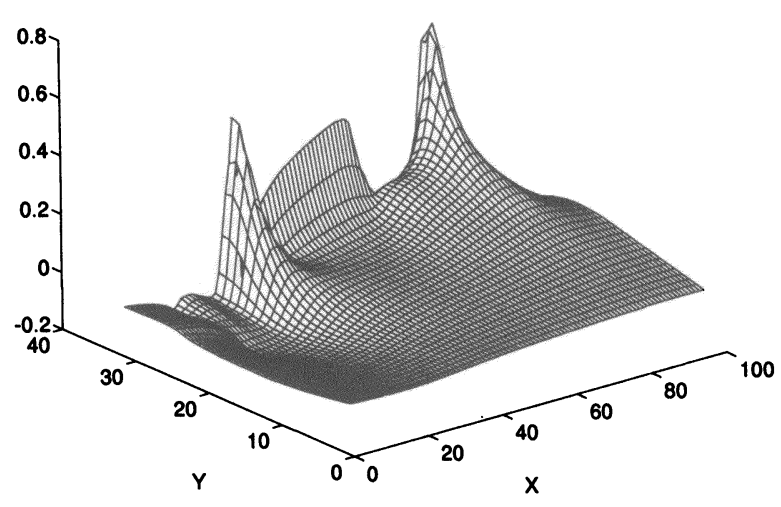

FIGURE 5A The $v_{y h}$ for the $96 \times 32$ mesh: the $\mathrm{P}^{1}$-RKGD method.

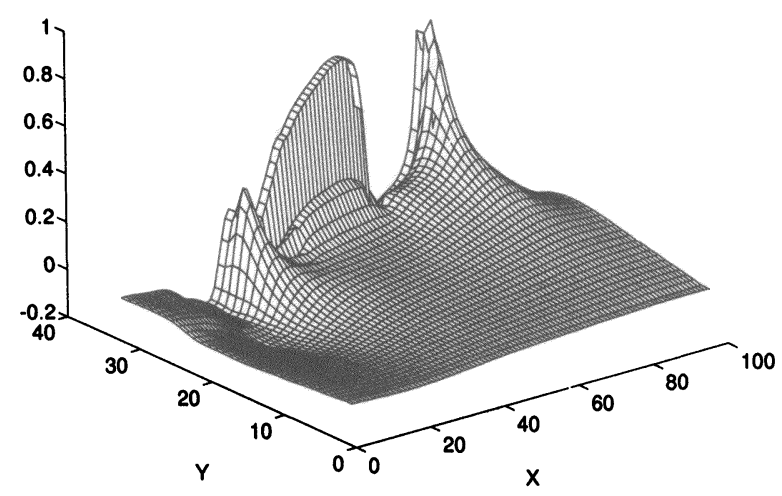

FIGURE 5B The $v_{y h}$ for the $96 \times 32$ mesh: the ENO method.

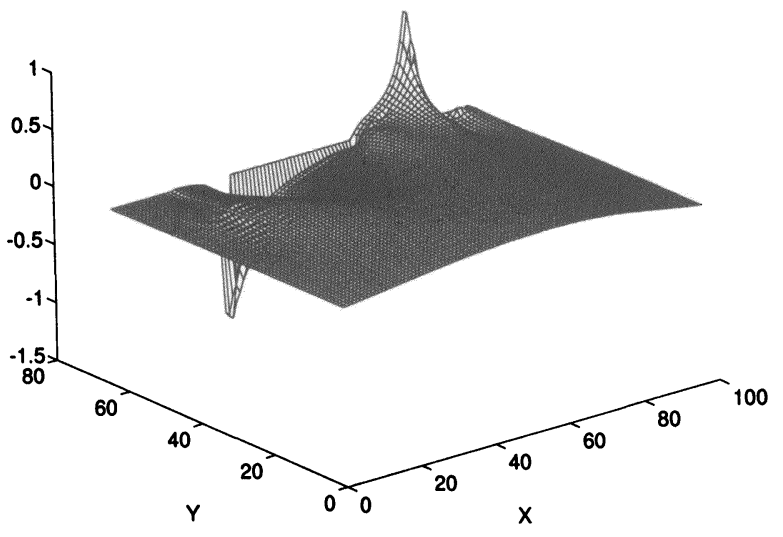

FIGURE 6B The $v_{x h}$ for the $192 \times 64$ mesh: the ENO method.

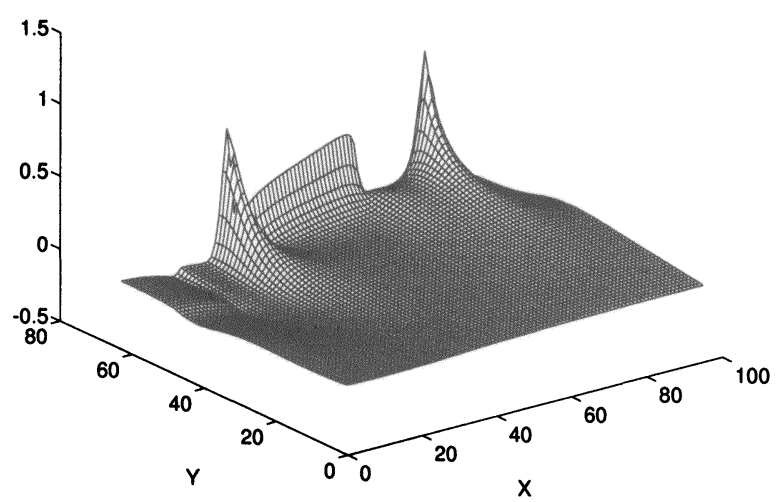

FIGURE 7A The $v_{y h}$ for the $192 \times 64$ mesh: the $\mathrm{P}^{1}-\mathrm{RKGD}$ method.

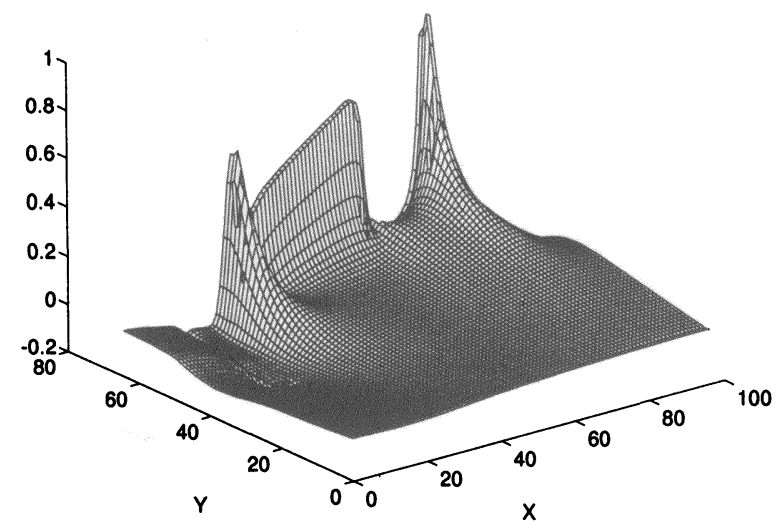

FIGURE 7B The $v_{y h}$ for the $192 \times 64$ mesh: the ENO method. method.

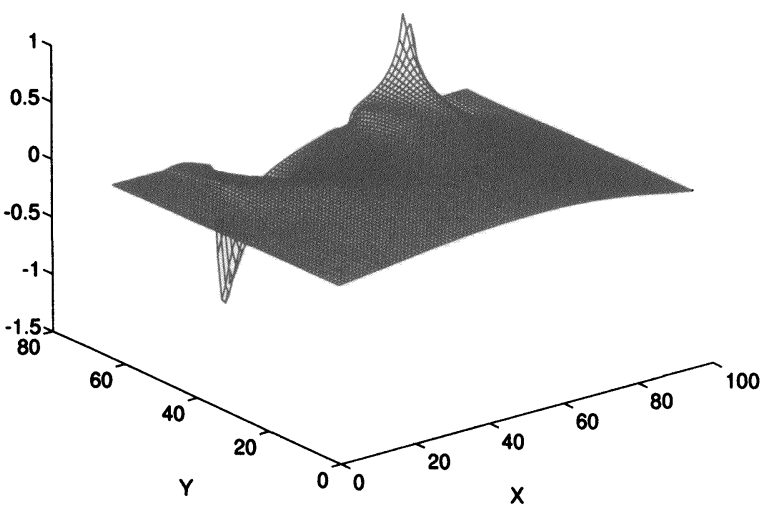

FIGURE 6A The $v_{x h}$ for the $192 \times 64$ mesh: the $\mathrm{P}^{1}$-RKGD 
the cusps and strong gradients in the components of the velocity. The junction layers and the interface layers are also clearly visible in the energy density $w$ and the potential $\phi$. The peaks of the electric field are due to its singularities around the intersections of the Dirichlet and Neumann segments.

To obtain an idea of the quality of the approximations, we compare the results of the RKGD method with piecewise linear approximate solutions with the results of the ENO method. We display surfaces of the components of the velocity in Figs. 4 and 5 (mesh $96 \times 32$ ) and in Figs. 6 and 7 (mesh $192 \times 64$ ). Only the components of the velocities are displayed, since the differences in the other quantities are not as noticeable. Cuts at $y=.1$ and at $y=.175$ of the density, temperature, and velocity are displayed in Figs. 8 and 9 (for the mesh $96 \times 32$ ) and on Figs. 10 and 11 (for the mesh $192 \times 64$ ). We can see that the results given by the $\mathrm{P}^{1}-\mathrm{RKDG}$ method are comparable to those of ENO; moreover, as we refine the
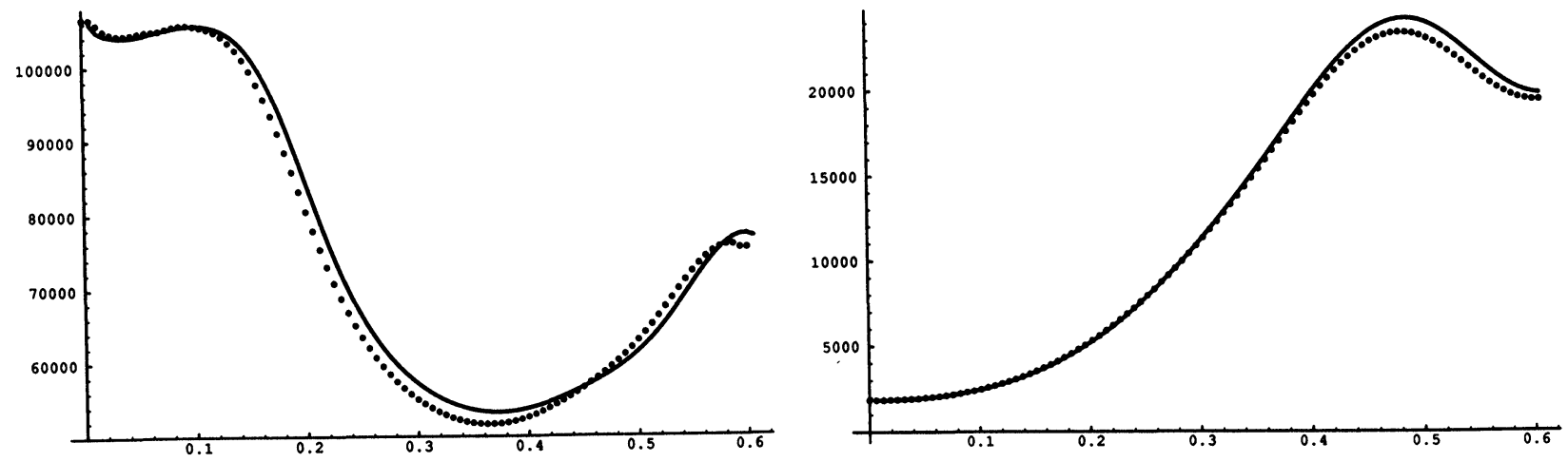

FIGURE 8A Cut of density (left) and temperature (right) at $y=.1$ for the $96 \times 32$ mesh. (-: P $^{1}$-RKDG, $\cdots:$ ENO)
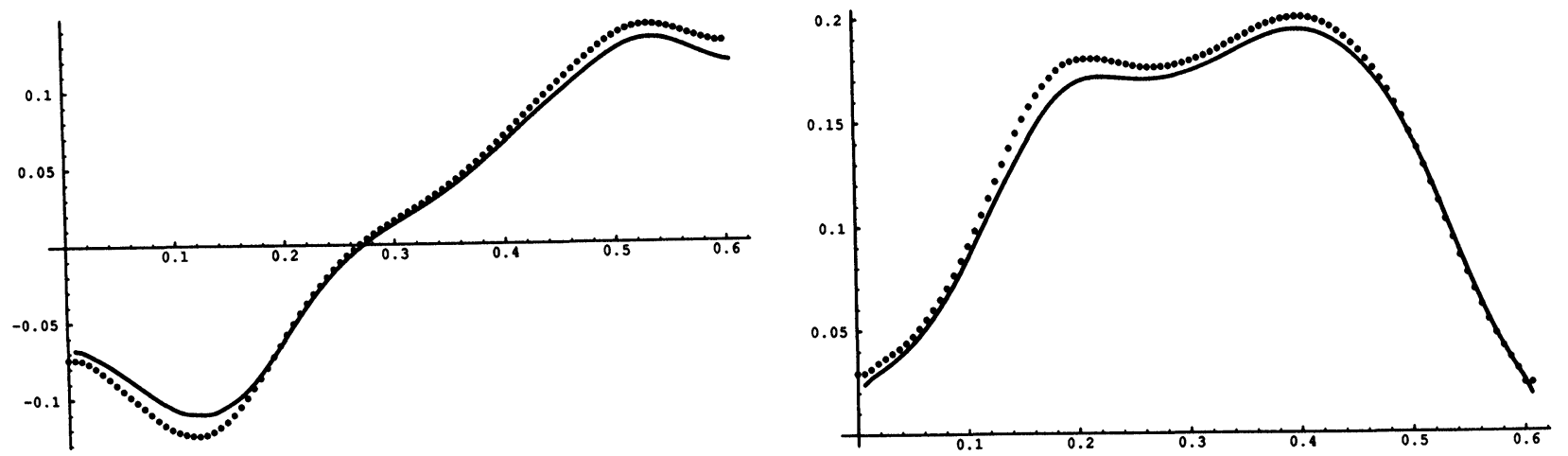

FIGURE 8B Cut of $v_{x h}$ (left) and $v_{y h}$ (right) at $y=.1$ for the $96 \times 32$ mesh. (-: $\mathrm{P}^{1}-\mathrm{RKDG}, \cdots:$ ENO)
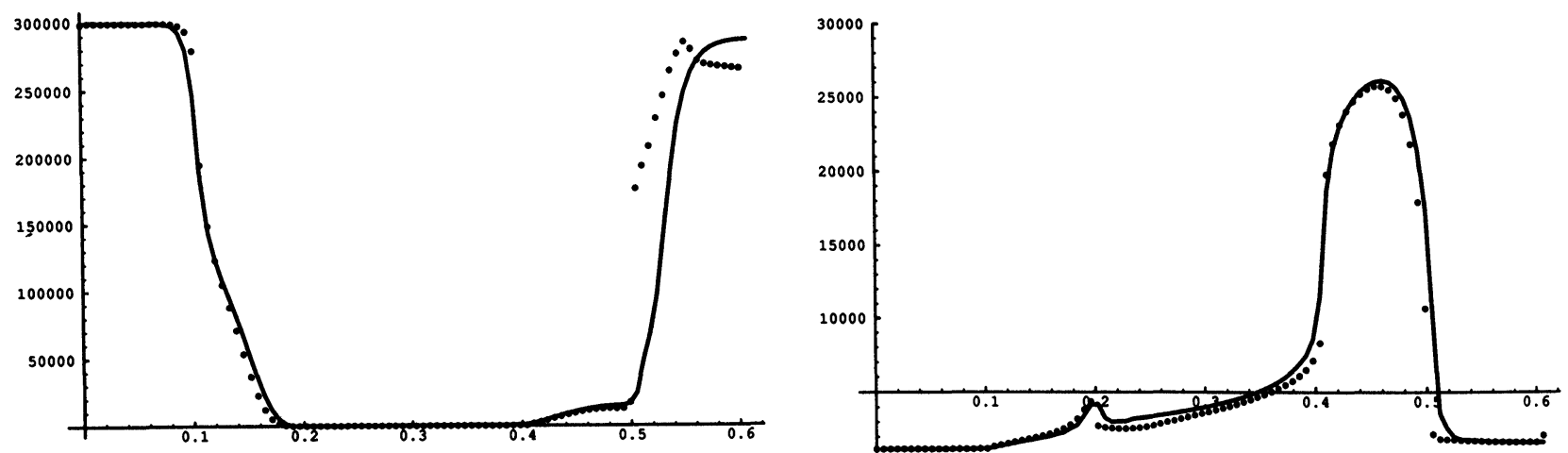

FIGURE 9A Cut of density (left) and temperature (right) at $y=.175$ for the $96 \times 32$ mesh. (-: $\mathrm{P}^{1}$-RKDG, $\cdots:$ ENO) 

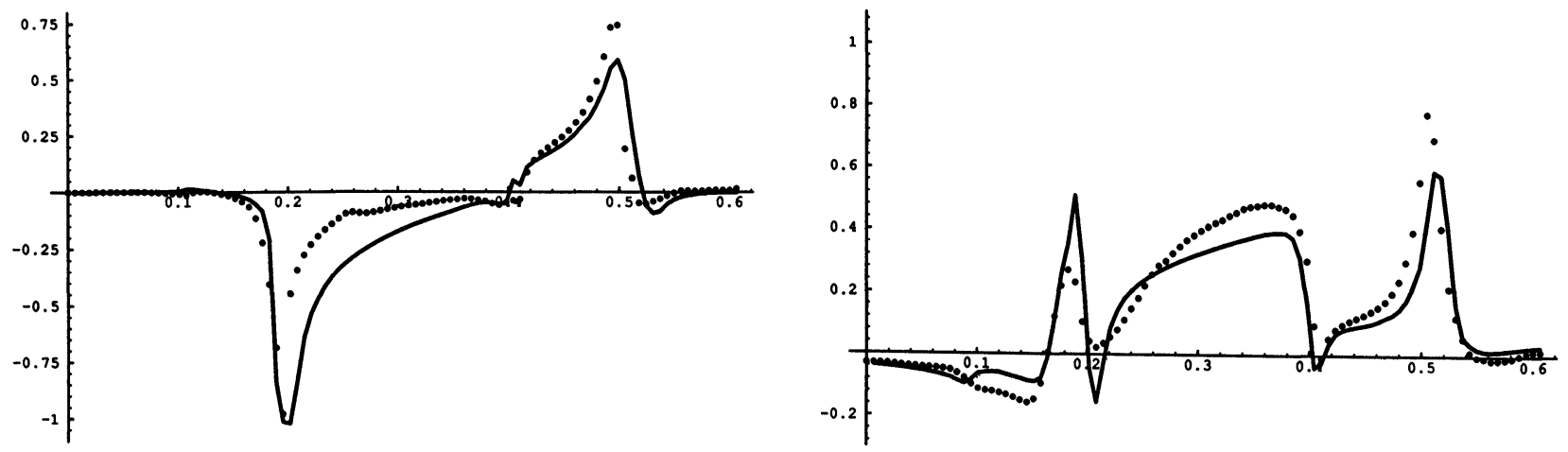

FIGURE 9B Cut of $v_{x h}$ (left) and $v_{y h}$ (right) at $y=.175$ for the $96 \times 32$ mesh. ( $-: \mathrm{P}^{1}$-RKDG, $\cdots:$ ENO)
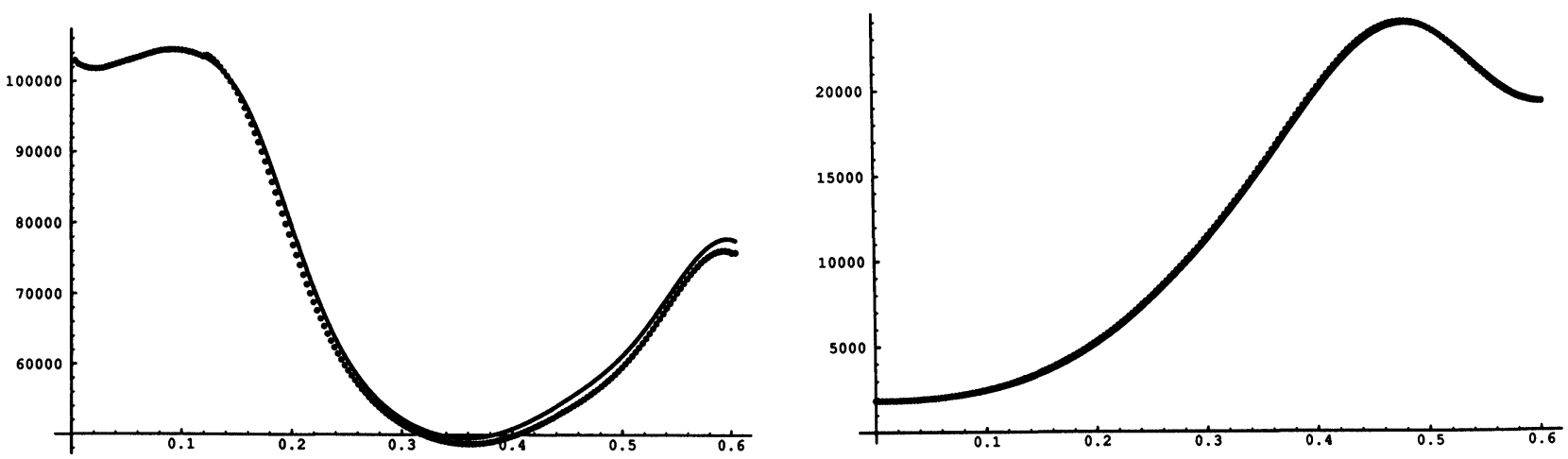

FIGURE 10A Cut of density (left) and temperature (right) at $y=.1$ for the $192 \times 62$ mesh. (一: $\mathrm{P}^{1}$-RKDG, $\cdots:$ ENO)
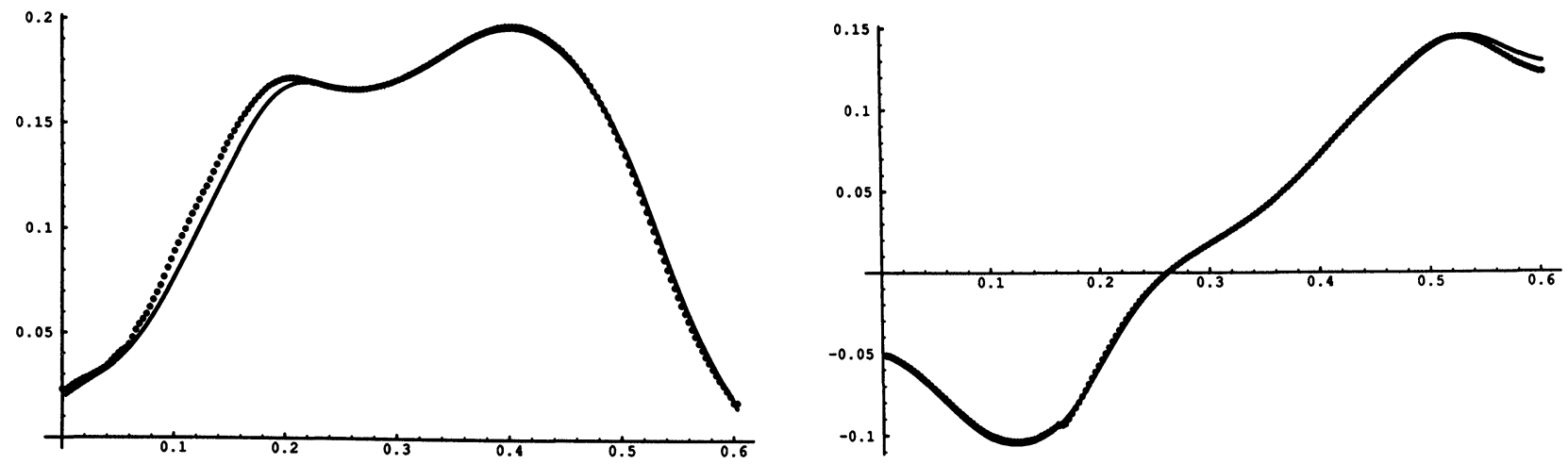

FIGURE 10B Cut of $v_{x h}$ (left) and $v_{y h}$ (right) at $y=.1$ for the $192 \times 64$ mesh. $\left(-: \mathrm{P}^{1}-\mathrm{RKDG}, \cdots:\right.$ ENO)
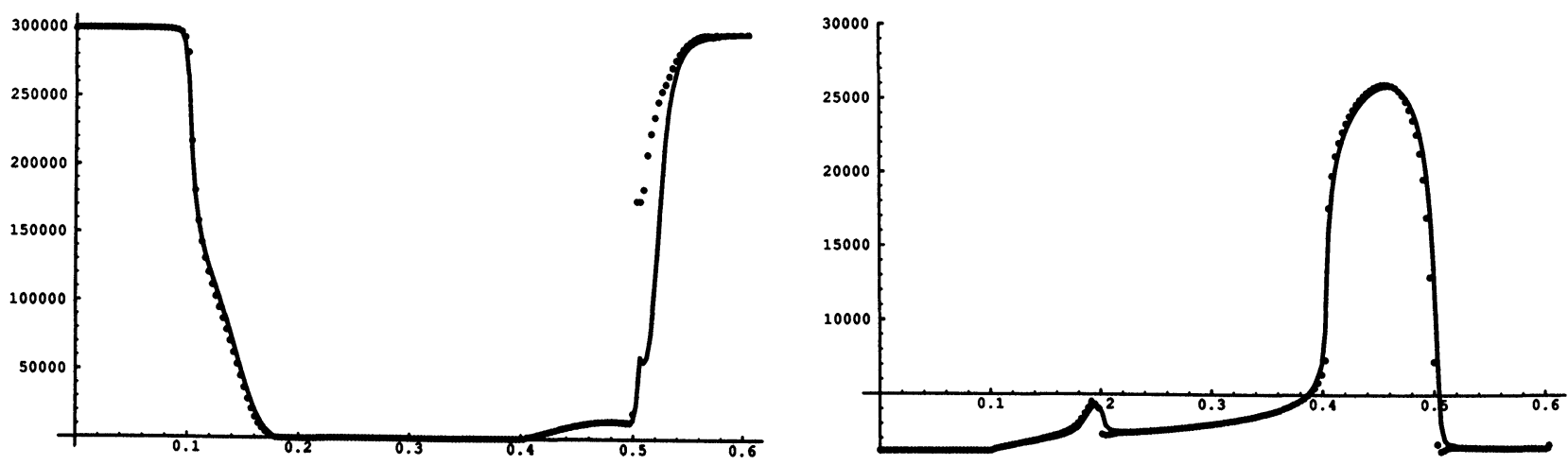

FIGURE 11A Cut of density (left) and temperature (right) at $y=.175$ for the $192 \times 64$ mesh. (-: $\mathrm{P}^{1}$-RKDG, $\cdots:$ ENO) 

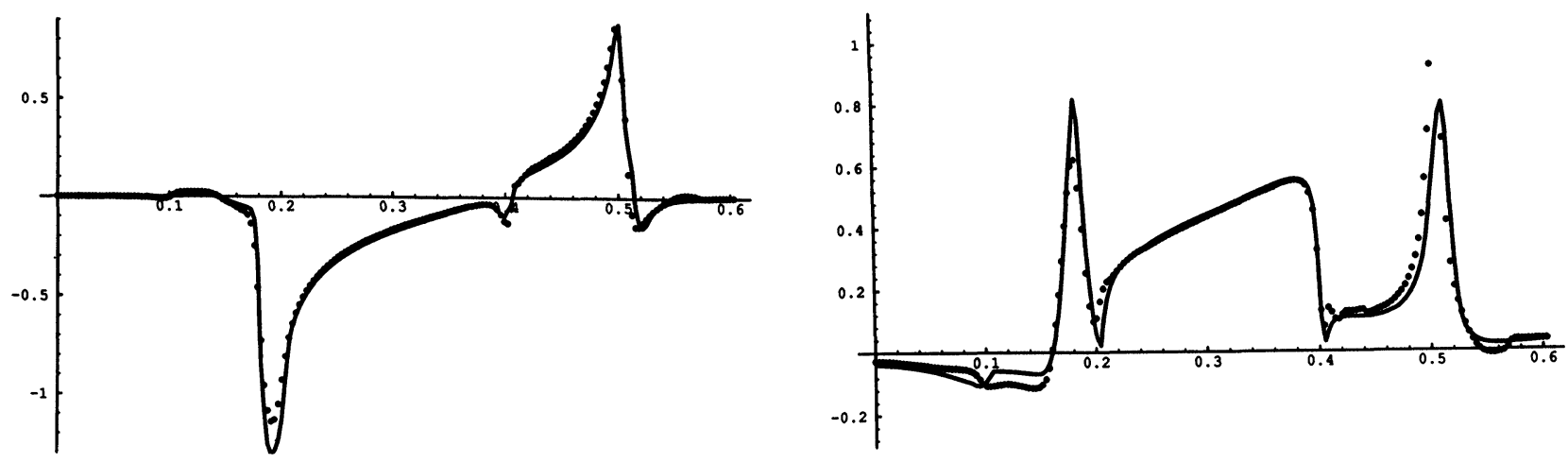

FIGURE 11B Cut of $v_{x h}$ (left) and $v_{y h}$ (right) at $y=.175$ for the $192 \times 64$ mesh. (-: $\mathrm{P}^{1}-\mathrm{RKDG}, \cdots:$ ENO)

mesh, we can see that these results get closer, as expected. There are noticeable differences in $v_{x h}$ and $v_{y h}$ along the gate between these two methods. The differences stem from the fact that the $P^{1}$ RKDG method treats the boundary conditions weakly, while the ENO method treats them strongly. Our numerical experiments (not reported here) also show that the approximation given by the $\mathrm{P}^{0}$-RKDG method, which is formally first-order accurate, is by far inferior to that provided by the $P^{1}-R K D G$ method, which is formally second-order accurate.

\section{CONCLUDING REMARKS}

The generalization of the RKDG methods to the two-dimensional hyperbolic system of conservation laws works very well. The performance of this formally second-order accurate method in uniform Cartesian grids is similar to that of the finite difference ENO method. In a future work, we plan to devise acceleration-to-the-steady-state techniques and to exploit the ability of our method to handle general triangulations.

\section{Acknowledgments}

The research of the first author is partly supported by the Army Research Office under contract DAAL03-89-C-0038 with the University of Minnesota Army High Performance Computing Research Center and by the Department of Energy under contract DE-ACOS-840R21400. The second author is partly supported by the National Science Foundation grant DSM-9103997, by the Minnesota Supercomputing Institute, and by the University of Minnesota Army High Performance Computing Research Center. The third author is partly supported by the National Science Foundation grant DMS-91232088. The final author is partly supported by the Army Research Office grant DAAL0391-G-0123 and the National Science Foundation grant ECS9214488.

\section{References}

[1] G. Baccarani and M.R. Wordeman, An investigation of steady-state velocity overshoot effects in $\mathrm{Si}$ and $\mathrm{GaAs}$ devices, Solid State Electr. 28 (1985), 407-416.

[2] F.J. Blatt, Physics of Electric Conduction in Solids, McGraw Hill, New York, 1968.

[3] F. Brezzi, J. Douglas, Jr., and L.D. Marini, Two families of mixed finite elements for second order elliptic problems, Numer. Math. 47 (1985), 217-235.

[4] B. Cockburn and C.W. Shu, TVB Runge-Kutta local projection discontinuous Galerkin finite element method for scalar conservation laws II: general framework, Math. Comp. 52 (1989), 411-435.

[5] B. Cockburn and C.W. Shu, The Runge-Kutta local projection $P^{1}$-discontinuous Galerkin finite element method for scalar conservation laws, $M^{2}$ AN25 (1991), 337-361.

[6] B. Cockburn, S.Y. Lin and C.W. Shu, TVB Runge-Kutta local projection discontinuous Galerkin finite element method for conservation laws III: one dimensional systems, J. Comput. Phys. 84 (1989), 90-113.

[7] B. Cockburn, S. Hou, and C.W. Shu, TVB Runge-Kutta local projection discontinuous Galerkin finite element method for conservation laws IV: the multidimensional case, Math. Comp. 54 (1990), 545-581.

[8] B. Cockburn and C.W. Shu, The $P^{1}-$ RKDG method for two-dimensional Euler equations of gas dynamics, ICASE Report No. 91-32 (1991).

[9] J. Douglas, Jr. and J. Roberts, Global estimates for mixed methods for second order elliptic problems, Math. Comp. 45 (1985), 39-52.

[10] E. Fatemi, J. Jerome, and S. Osher, Solution of the hydrodynamic device model using high-order nonoscillatory shock capturing algorithms, IEEE Transactions on Computer Aided Design of Integrated Circuits and Systems CAD-10 (1991), 232-244.

[11] E. Fatemi, C. Gardner, J. Jerome, S. Osher, and D. Rose, Simulation of a steady-state electron shock wave in a submicron semiconductor device using high-order upwind methods, Computational Electronics, K. Hess, J.P. Leburton, and U. Ravaioli, ed., Kluwer, Boston, 1991, pp. 27-32.

[12] C.L. Gardner, Numerical simulation of a steady-state electron shock wave in a submicrometer semiconductor device, IEEE Transactions on Elèctron Devices ED-38 (1991), 392-398.

[13] C.L. Gardner, Hydrodynamic and Monte-Carlo simulation of an electron shock wave in a $1-\mu \mathrm{m} n^{+}-n-n^{+}$diode, IEEE Transactions on Electron Devices ED-40 (1993), 455-457.

[14] W. Hänsch and M. Miura-Mattausch, The hot-electron problem in small semiconductor devices, J. Appl. Physics 60 (1986), 650-656. 
[15] J. Jerome and C.-W. Shu, Energy models for one-carrier transport in semiconductor devices, to appear in IMA Volumes in Mathematics and its Applications, F. Odeh et al., editors, Springer-Verlag.

[16] J. Nougier, J. Vaissiere, D. Gasquet, J. Zimmermann, and E. Constant, Determination of the transient regime in semiconductor devices using relaxation time approximations. J. Appl. Physics 52 (1981), 825-832.

[17] P.A. Raviart and J.M. Thomas, A mixed finite element method for second order elliptic problems, Lecture Notes in Math. 606, Springer, Berlin, 1977, pp. 292-315.

[18] C.-W. Shu and S. Osher, Efficient implementation of essentially non-oscillatory shock capturing schemes, J. Comp. Phys. 77 (1988), 439-471.

[19] C.-W. Shu and S. Osher, Efficient implementation of essentially non-oscillatory shock capturing schemes, II, J. Comp. Phys. 83 (1989), 32-78.

[20] C.-W. Shu, T. Zang, G. Erlebacher, D. Whitaker, and S. Osher, High-order ENO schemes applied to two- and three-dimensional compressible flow, J. Appl. Numer. Math. 9 (1991), 45-71.

\section{Biographies}

ZHANGXIN CHEN is an assistant professor in Department of Mathematics and the Institute for Scientific Computation, Texas
A \& M University. His research interests include numerical partial differential equations, scientific computing, and mathematical modeling. He is a member of AMS and SIAM. He received the $\mathrm{Ph} . \mathrm{D}$. degree in mathematics from Purdue University, and spent two years as a post-doctoral fellow at University of Minnesota.

BERNARDO COCKBURN received the Ph.D. degree in Mathematics from the University of Chicago, Chicago, IL, in 1986. He is currently an Associate Professor at the School of Mathematics, University of Minnesota. Previously, he was a post-doc at the Institute for Mathematics and its Applications, and an Assistant Professor at the School of Mathematics, University of Minnesota. His current interests lie in theoretical and practical aspects of the numerical analysis classical conservation laws, semiconductor device simulation, and phase propagation in solids.

JOSEPH W. JEROME received the Ph.D. degree in Mathematics from Purdue University in 1966 . He was visiting Assistant Professor at the Mathematics Research Center, University of Wisconsin, during 1966-68, and was Assistant Professor at Case Western Reserve University during 1968-70. He joined Northwestern University in 1970, where he has been Professor of Mathematics and Applied Mathematics since 1976. He has held sabbatical positions at Oxford University, England, 1974-75, University of Texas, 1978-79, and Bell Laboratories, Murray Hill, 1982-83. He was visiting scholar at the University of Chicago in 1985. His research interests include applied and numerical analysis, computational electronics, and cell membrane biology. His book on semiconductor modeling will be published by Springer in 1995 . 

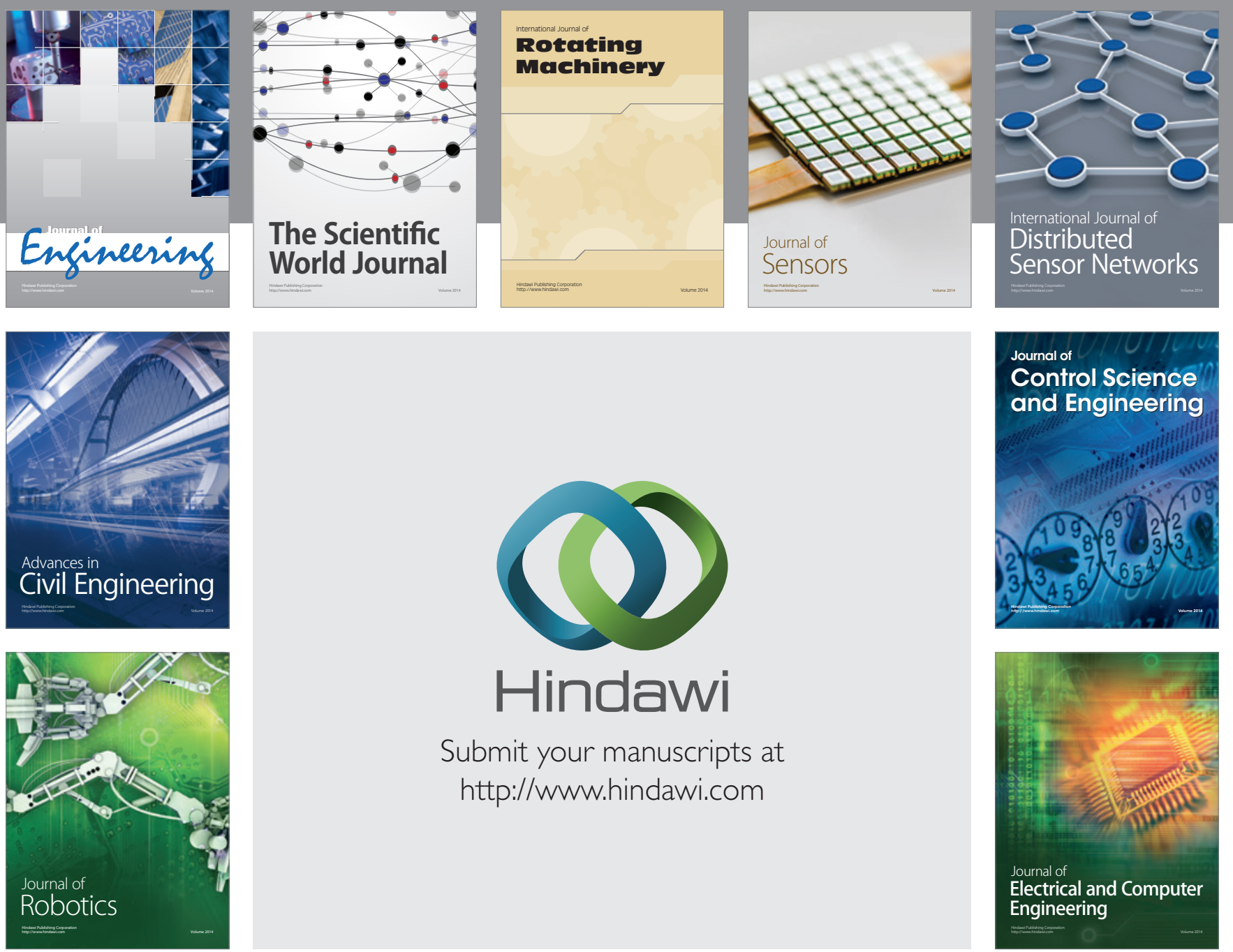

Submit your manuscripts at

http://www.hindawi.com
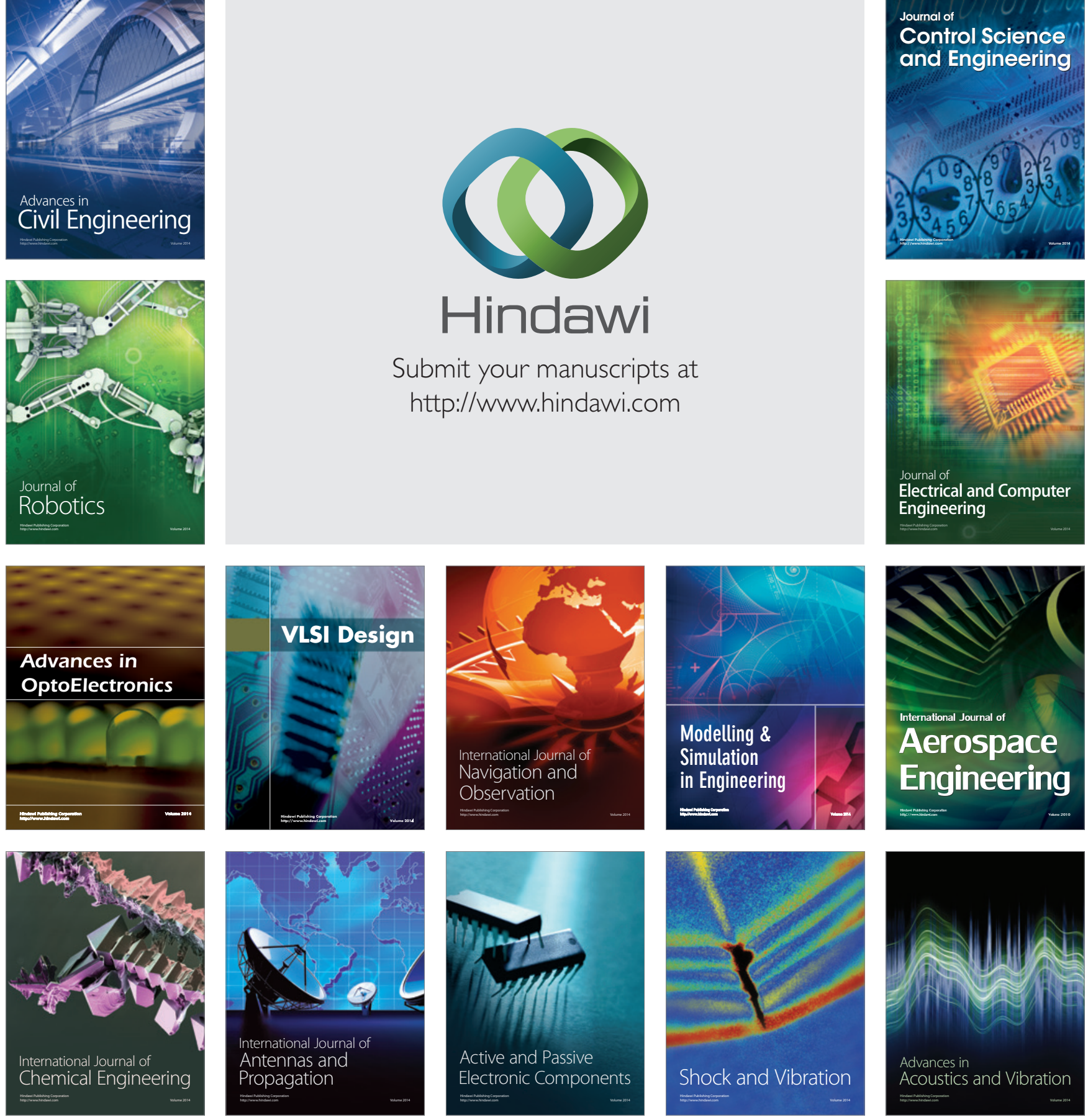